\title{
Existence and $p$-exponential stability of periodic solution for stochastic shunting inhibitory cellular neural networks with time-varying delays
}

\author{
Changjin $\mathrm{Xu}^{1 *}$, Maoxin Liao ${ }^{2}$, Yicheng Pang ${ }^{3}$ \\ ${ }^{1}$ Guizhou Key Laboratory of Economics System Simulation \\ Guizhou University of Finance and Economics, \\ Guiyang 550004, PR China \\ E-mail:xcj403@126.com \\ ${ }^{2}$ School of Mathematics and Physics, University of South China, \\ Hengyang 421001, PR China \\ E-mail:maoxinliao@163.com \\ ${ }^{3}$ School of Mathematics and Statistics, Guizhou University of Finance and Economics, \\ Guiyang 550004, PR China \\ E-mail: ypanggy@outlook.com
}

Received 31 October 2014

Accepted 20 June 2016

\begin{abstract}
In this paper, we investigate a class of stochastic shunting inhibitory cellular neural networks with time-varying delays. Applying integral inequality, some sufficient conditions on the existence and $p$ exponential stability of periodic solutions for stochastic shunting inhibitory cellular neural networks with time-varying delays are established. An example is presented to illustrate our main theoretical findings. Our results are new and complementary to previously known studies.
\end{abstract}

Keywords: Stochastic shunting inhibitory cellular neural networks, periodic solution, $p$-exponential stability, delay.

\section{Introduction}

Since shunting inhibitory cellular neural networks have been successfully applied to pattern recognition, image and signal processing, vision, and optimization, their dynamics has attracted many attentions. Numerous important results on the existence and uniqueness of equilibrium point, periodic solution, almost periodic solution, pseudo almost periodic solution, almost automorphic solution and antiperiodic solution have been reported. For example,
Gao et al. ${ }^{1}$ studied the existence and stability almost periodic solutions for cellular neural networks with time-varying delays in leakage terms on time scales, Liu and $\mathrm{Shao}^{2}$ analyzed the almost periodic solutions for SICNNs with time-varying delays in the leakage terms, $\mathrm{Liu}^{3}$ focused on the pseudo almost periodic solutions for neutral type CNNs with continuously distributed leakage delays, Armmaret al. ${ }^{4}$ made a detailed discussion on the existence and uniqueness of pseudo almost periodic solutions of recurrent neural networks with time-varying coef-

\footnotetext{
* Corresponding author, Email:xcj403@126.com(C.Xu).
} 
ficients and mixed delays, Abbas and Xia ${ }^{5}$ investigated the almost automorphic solutions of impulsive cellular neural networks with piecewise constant argument, $\mathrm{Li}$ and $\mathrm{Shu}{ }^{6}$ dealt with the antiperiodic solutions to impulsive shunting inhibitory cellular neural networks with distributed delays on time scales. For details, we refer readers to papers $7,8,9,10,11,12,13,14$.

In 1994, Haykin ${ }^{15}$ pointed out that in real nervous systems, synaptic transmission is a noisy process brought on by random fluctuations from the release of neurotransmitters and other probabilistic causes. Neural networks could be stabilized or destabilized by some stochastic inputs ${ }^{16}$. Considering that neural networks are inevitably affected by the random fluctuations from the release of neurotransmitters and other probabilistic causes which is an important component in neural networks, we think that it is worth while to investigate the stochastic neural networks. Recently, there are many papers that deal with this aspect ${ }^{17,18,19,20,21}$. In this paper, we will consider the following stochastic shunting inhibitory cellular neural networks with timevarying delays

$$
\begin{aligned}
& d x_{i j}(t)=\left[-a_{i j}(t) x_{i j}(t)+L_{i j}(t)\right. \\
& -\sum_{B^{k l} \in N_{r}(i, j)} B_{i j}^{k l}(t) f_{i j}\left(t, x_{k l}(t)\right) x_{i j}(t) \\
& \left.-\sum_{C^{k l} \in N_{r}(i, j)} C_{i j}^{k l}(t) g_{i j}\left(t, x_{k l}\left(t-\tau_{k l}(t)\right)\right) x_{i j}(t)\right] d t \\
& +\sum_{D^{k l} \in N_{r}(i, j)} D_{i j}^{k l}(t) \sigma_{i j}\left(x_{i j}(t)\right) d w_{i j}(t), t \geqslant t_{0}, \quad(1)
\end{aligned}
$$

where $i=1,2, \cdots, m, j=1,2, \cdots, n, \tau_{i j}(t)>0$ denotes axonal signal transmission delay at time $t$, $C_{i j}(t)$ denotes the cell at the $(i, j)$ position of the lattice at time $t$, the $r$-neighborhood $N_{r}(i, j)$ of $C_{i j}(t)$ is given as $N_{r}(i, j)=\left\{C_{i j}^{k l}: \max (|k-i|,|l-j| \leqslant r), 1 \leqslant\right.$ $k \leqslant m, 1 \leqslant l \leqslant n\}, x_{i j}(t)$ stands for the activity of the cell $C_{i j}(t), L_{i j}(t)$ denotes the external input to $C_{i j}(t), a_{i j}(t)>0$ stands for the passive decay rate of the cell activity, $B_{i j}^{k l}(t) \geqslant 0$ and $C_{i j}^{k l}(t) \geqslant 0$ represent the connection or coupling strength of postsynaptic of activity of the cell transmitted to the cell $C_{i j}(t)$ at time $t$ and $t-\tau_{k l}(t)$, respectively, the activity functions $f_{i j}\left(t, x_{k l}(t)\right)$ and $g_{i j}\left(t, x_{k l}(t)\right)$ are con- tinuous functions representing the output or firing rate of the cell $C_{k l}(t)$ at time $t$ and $t-\tau_{k l}(t)$, respectively, $w(t)=\left(w_{11}(t), w_{12}(t), \cdots, w_{m n}(t)\right)^{T}$ is $m \times n$ dimensional Brownian motions defined on a complete probability space, $\sigma_{i j} \in C(R, R)$ is a Borel measurable function and $\sigma=\left(\sigma_{i j}\right)_{m n \times m n}$ is a diffusion coefficient matrix.

Let $R^{n}\left(R_{+}^{n}\right)$ be the space of $n$-dimensional (nonnegative) real column vectors and $R^{m n}$ be the space of $m \times n$-dimensional real column vectors. We denote $\left(\Omega, F,\left\{F_{t}\right\}_{t \geqslant 0}, P\right)$ by a complete probability space with a filtration $\left\{F_{t}\right\}_{t \geqslant 0}$, where $F$ is a $\sigma$-algebra on a given set $\Omega, P$ is the probability measure and the filtration Ftt. satisfies the usual conditions, that is, $\left\{F_{t}\right\}_{t \geqslant 0}$ is right continuous and $F_{0}$ contains all $P$-null sets. Denote by $B C_{F_{0}}^{b}\left(R, R^{m n}\right)$ the family of bounded $F_{0}$-measurable, $R^{m n}$ valued random variables $x(t)$, that is, the value of $x(t)$ is an $m \times n$-dimensional real vector and can be decided from the values of $w(s)$ for $s \leqslant$ 0. Then $B C_{F_{0}}^{b}\left(R, R^{m n}\right)$ is a Banach space with the norm $\|x\|=\sup _{0 \leqslant t \leqslant \omega}\left(E|x(t)|_{1}^{p}\right)^{\frac{1}{p}}$, where $p>$ 1 is an integer, $|x(t)|_{1}=\max _{(i, j)}\left|x_{i j}(t)\right|$, and $E($. stands for the correspondent expectation operator with respect to the given probability measure $P$. For convenience, for an $\omega$-periodic continuous function $f: R \rightarrow R$, denote $f=\max _{0 \leqslant t \leqslant \omega}|f(t)|, f=$ $\min _{0 \leqslant t \leqslant \omega}|f(t)|$, for any $\phi \in B C_{F_{0}}^{b}\left([-\tau, 0], R^{m n}\right)$, denote $[\phi(t)]_{\tau}^{+}=\left(\left|\phi_{11}\right|_{\tau},\left|\phi_{12}\right|_{\tau}, \cdots,\left|\phi_{m n}\right|_{\tau}\right)^{T}$, where $\left|\phi_{i j}\right|_{\tau}=\sup _{-\tau \leqslant s \leqslant 0}\left|\phi_{i j}(t+s)\right|, i=1,2, \cdots, m, j=$ $1,2, \cdots, n$.

The initial value of system (1) takes the form

$$
x_{i j}(s)=\varphi_{i j}(s), s \in\left[t_{0}-\tau, t_{0}\right],
$$

where $\varphi_{i j}(s) \in B C_{F_{0}}^{b}\left(\left[t_{0}-\tau, 0\right], R\right), \tau=$ $\max _{1 \leqslant k \leqslant m, 1 \leqslant l \leqslant n}\left\{\tau_{k l}\right\}, t_{0} \in R$.

Throughout this paper, we make the assumption as follows.

(H1) For $i=1,2, \cdots, m, j=1,2, \cdots, n$, $a_{i j}(t), B_{i j}^{k l}(t), C_{i j}^{k l}(t), D_{i j}^{k l}(t)$ and $L_{i j}(t)$ are all $\omega$ periodic continuous functions for all $t \in R$.

(H2) For $i=1,2, \cdots, m, j=1,2, \cdots, n$, there exist positive constants $L_{i j f}, g_{i j g}, \sigma_{i j \sigma}, M_{f}$ and $M_{g}$ such 
that

$$
\begin{aligned}
& \left|f_{i j}(t, u)-f_{i j}(t, v)\right| \leqslant L_{i j f}|u-v|,\left|f_{i j}(u)\right| \leqslant M_{f}, \\
& \left|g_{i j}(t, u)-g_{i j}(t, v)\right| \leqslant L_{i j g}|u-v|,\left|g_{i j}(u)\right| \leqslant M_{g}, \\
& \left|\sigma_{i j}(t, u)-\sigma_{i j}(t, v)\right| \leqslant L_{i j \sigma}|u-v|
\end{aligned}
$$

for all $u, v, t \in R$.

The remainder of the paper is organized as follows: in Section 2, several definitions and some preliminary results which are useful in later section are introduced. some sufficient conditions for the the existence of periodic solutions of system (1) are derived in Section 3. In Section 4, the p-exponential stability of periodic solutions are analyzed. An examples are given to illustrate the feasibility and effectiveness of our results obtained in previous section in Section 5. A brief conclusion is drawn in Section 6.

\section{Preliminaries}

In this section, we shall recall several definitions and present some preliminary results which are necessary in later sections.

Definition 2.1 ${ }^{22}$ A stochastic process $x(t)$ is said to be periodic with period $\omega$ if its finite-dimensional distributions are periodic with period $\omega$, that is, for any positive integer $m$ and any moments of time $t_{1}, t_{2}, \cdots, t_{m}$, the joint distribution of the random variables $\left.x\left(t_{1}+k \omega\right), x\left(t_{2}+k \omega\right), \cdots, x\left(t_{m}+k \omega\right)\right)$ are independent of $k, k= \pm 1, \pm 2, \cdots$.

Lemma $2.1{ }^{23}$ If $x(t)$ is an $\omega$-periodic stochastic process, then its mathematical expectation and variance are $\omega$-periodic.

Definition 2.2 A function $x(t)=\left(x_{11}(t), x_{12}(t), \cdots\right.$, $\left.x_{m n}(t)\right)^{T}$ defined on $\left[t_{0}-\tau, \infty\right)$ is said to be a solution of (1) with initial condition (2) if the following conditions holds.

(i) $x_{i j}(t)$ is absolutely continuous on $\left[t_{0}-\tau, \infty\right), i=$ $1,2, \cdots, m, j=1,2, \cdots, n$,

(ii) $x_{i j}(t)$ satisfies (1) for almost everywhere $t \in$ $\left[t_{0}, \infty\right), i=1,2, \cdots, m, j=1,2, \cdots, n$,

(iii) $x_{i j}(s)=\varphi_{i j}(s), s \in\left[t_{0}-\tau, t_{0}\right], i=1,2, \cdots, m, j=$ $1,2, \cdots, n$.
Throughout this paper, we assume that (1) with initial condition (2) has a unique solution. Denote the solution of (1) by $x(t)=x\left(t, t_{0}, \varphi\right)$ for all $\varphi \in$ $B C_{F_{0}}^{b}\left(\left[t_{0}-\tau, t_{0}\right], R^{m n}\right)$ and $t_{0} \in R$.

Definition $2.3^{22}$ The solution $x\left(t, t_{0}, \varphi\right)$ of $(1)$ is said to be

(i) p-uniformly bounded, if for each $\alpha>0, t_{0} \in R$, there exists a positive constant $\theta=\theta(\alpha)$ which is independent of $t_{0}$ such that $\|\varphi\|^{p} \leqslant \alpha$ implies $E\left(x\left(t, t_{0}, \varphi\right) \|^{p}\right) \leqslant \theta, t \geqslant t_{0}$;

(ii) p-point dissipative, if there exists a constant $N>0$ such that for any point $\varphi \in B C_{F_{0}}^{b}\left([-\tau, 0], R^{n}\right)$, there exists $T\left(t_{0}, \varphi\right)$ such that $E\left(\left\|x\left(t, t_{0}, \varphi\right)\right\|^{p}\right) \leqslant$ $N, t \geqslant t_{0}+T\left(t_{0}, \varphi\right)$.

Lemma 2.2 ${ }^{24}$ In addition to (H1) and (H2), suppose that the solution of (1) is p-uniformly bounded and p-point dissipative for $p>2$, then (1) has an $\omega$-periodic solution.

Lemma $2.3^{25}$ For any $x \in R_{+}^{n}$ and $p>0$,

$$
|x|^{p} \leqslant n^{\left(\frac{p}{2}-1\right) \vee 0} \sum_{i=1}^{n} x_{i}^{p},\left(\sum_{i=1}^{n} x_{i}\right)^{p} \leqslant n^{(p-1) \vee 0} \sum_{i=1}^{n} x_{i}^{p} .
$$

Definition 2.4 ${ }^{22}$ The periodic solution $x\left(t, t_{0}, \varphi\right)$ with initial value $\varphi \in B C_{F_{0}}^{b}\left([-\tau, 0], R^{n}\right)$ of $(1)$ is said to be p-exponentially stable, if there are constants $\lambda>0$ and $M>0$ such that for any solution $y\left(t, t_{0}, \varphi_{1}\right)$ with initial value $\varphi_{1} \in B C_{F_{0}}^{b}\left([-\tau, 0], R^{n}\right)$ of (1) satisfies

$$
E\left(|x-y|_{1}^{p}\right) \leqslant M \mid \text { varphi }-\varphi_{1}||^{p} e^{-\lambda\left(t-t_{0}\right)}, t \geqslant t_{0} .
$$

Lemma 2.4 ${ }^{22}$ Let $u(t) \in C\left(R, R_{+}^{n}\right)$ be a solution of the delay integral inequality

$$
\left\{\begin{aligned}
u(t) \leqslant & M_{1} e^{-\delta\left(t-t_{0}\right)}[\varphi]_{\tau}^{+}+\int_{t_{0}}^{t} e^{-C_{1}(t-s)} A_{1} u(s) d s \\
& +\int_{t_{0}}^{t} e^{-C_{1}(t-s)} B_{1}[u(s)]_{\tau}^{+} d s+J_{1}, t \geqslant t_{0}, \\
u(t) & \leqslant \varphi(t), \forall t \in\left[t_{0}-\tau, t_{0}\right],
\end{aligned}\right.
$$

where $A_{1}, B_{1}, C_{1}, M_{1} \in R_{+}^{n \times n}, J_{1} \geqslant 0$ is a constant vector, $\varphi(t) \in C\left(\left[t-\tau, t_{0}\right], R_{+}^{n}\right)$. If $\rho(\Pi)<1$, where $\Pi=C_{1}^{-1}\left(A_{1}+B_{1}\right)$, then there are constants $0<\lambda \leqslant$ $\delta$ and $N \geqslant 1$ such that

$$
u(t) \leqslant N z e^{-\lambda\left(t-t_{0}\right)}+(I-\Pi)^{-1} J_{1}, t \geqslant t_{0},
$$


where z satisfies $[\varphi]_{\tau}^{+} \leqslant z$.

Lemma 2.5 22 Assume that all conditions of Lemma 2.4 hold. If $J_{1}=0$, then all solutions of inequality of (1) exponentially convergent to zero.

In view of Lemma 2.4 and Lemma 2.5, we have the following results.

Lemma 2.6 ${ }^{26}$ Let $u(t) \in C\left(R, R_{+}^{n}\right)$ be a solution of the delay integral inequality

$$
\left\{\begin{aligned}
u(t) & \leqslant M_{1} e^{-\delta\left(t-t_{0}\right)}[\varphi]_{\tau}^{+}+\int_{t_{0}}^{t} e^{-C_{1}(t-s)} A_{1} u(s) d s \\
& +\int_{t_{0}}^{t} e^{-C_{1}(t-s)} B_{1}[u(s)]_{\tau}^{+} d s+J_{1}, t \geqslant t_{0}, \\
u(t) & \leqslant \varphi(t), \forall t \in\left[t_{0}-\tau, t_{0}\right],
\end{aligned}\right.
$$

where $A_{1}, B_{1}, C_{1}, M_{1} \in R_{+}^{n \times n}, J_{1} \geqslant 0$ is a constant vector, $\varphi(t) \in C\left(\left[t-\tau, t_{0}\right], R_{+}^{n}\right)$. If $\frac{A_{1}+B_{1}}{C_{1}}<1$, then there are constants $0<\lambda \leqslant \delta$ and $N \geqslant 1$ such that

$$
u(t) \leqslant N z e^{-\lambda\left(t-t_{0}\right)}+\left(1-\frac{A_{1}+B_{1}}{C_{1}}\right)^{-1} J_{1}, t \geqslant t_{0},
$$

where $z$ satisfies $[\varphi]_{\tau}^{+} \leqslant z$. Moreover, if $J_{1}=0$, then all solutions of the inequality of (4) are exponentially convergent to zero.

\section{Existence of Periodic Solution}

In this section, we discuss the existence of periodic solution of (1).

Theorem 3.1 In addition to (H1) and (H2), assume further that

(H3) there exists an integer $p>2$ such that $\rho \delta^{-1}<$ 1 , where $\delta=\min _{(i, j)}\left\{\underline{a}_{i j}\right\}, \sigma=\left(\frac{p(p-1)}{2}\right)^{\frac{p}{2}}$,

$$
\begin{aligned}
\rho= & \max _{(i, j)}\left\{5 ^ { p - 1 } ( \underline { a } _ { i j } ) ^ { 1 - p } \left[\left(M_{f} \sum_{B^{k l} \in N_{r}(i, j)} \bar{B}_{i j}^{k l}\right)^{p}\right.\right. \\
& \left.+\left(M_{g} \sum_{C^{k l} \in N_{r}(i, j)} \bar{C}_{i j}^{k l}\right)^{p}\right] \\
& +\sigma(m n)^{\frac{p}{2}}\left(\frac{2 \underline{a}_{i j}(p-1)}{p-2}\right)^{1-\frac{p}{2}} \\
& \times\left(\sum_{D^{k l} \in N_{r}(i, j)} \bar{D}_{i j}^{k l}\right)^{p}
\end{aligned}
$$

then (1.1) has an $\omega$-periodic solution.

Proof It follows from the method of variation parameter and (1) that for $t \geqslant t_{0}, i=1,2, \cdots, m, j=$ $1,2, \cdots, n$,

$$
\begin{aligned}
& x_{i j}(t)=x_{i j}\left(t_{0}\right) e^{-\int_{t_{0}}^{t} a_{i j}(\xi) d \xi}-\int_{t_{0}}^{t} e^{-\int_{t_{0}}^{t} a_{i j}(\xi) d \xi} \\
& {\left[\sum_{B^{k l} \in N_{r}(i, j)} B_{i j}^{k l}(s) f_{i j}\left(s, x_{k l}(s)\right) x_{i j}(s)\right.} \\
& +\sum_{C^{k l} \in N_{r}(i, j)} C_{i j}^{k l}(s) g_{i j}\left(s, x_{k l}\left(s-\tau_{k l}(s)\right)\right) x_{i j}(s) \\
& \left.-L_{i j}(s)\right] d s+\int_{t_{0}}^{t} e^{-\int_{t_{0}}^{t} a_{i j}(\xi) d \xi} \\
& \sum_{D^{k l} \in N_{r}(i, j)} D_{i j}^{k l}(s) \sigma_{i j}\left(x_{i j}(s)\right) d w_{i j}(s) .
\end{aligned}
$$

Let

$$
\begin{aligned}
& \Theta_{i j}^{(1)}=x_{i j}\left(t_{0}\right) e^{-\int_{t_{0}}^{t} a_{i j}(\xi) d \xi}, \\
& \Theta_{i j}^{(2)}=\int_{t_{0}}^{t} e^{-\int_{t_{0}}^{t} a_{i j}(\xi) d \xi} \\
& \sum_{B^{k l} \in N_{r}(i, j)} B_{i j}^{k l}(s) f_{i j}\left(s, x_{k l}(s)\right) x_{i j}(s) d s, \\
& \Theta_{i j}^{(3)}=\int_{t_{0}}^{t} e^{-\int_{t_{0}}^{t} a_{i j}(\xi) d \xi} \sum_{C^{k l} \in N_{r}(i, j)} C_{i j}^{k l}(s) \\
& \times g_{i j}\left(s, x_{k l}\left(s-\tau_{k l}(s)\right)\right) x_{i j}(s) d s, \\
& \Theta_{i j}^{(4)}=\int_{t_{0}}^{t} e^{-\int_{t_{0}}^{t} a_{i j}(\xi) d \xi} L_{i j}(s) d s, \\
& \Theta_{i j}^{(5)}=\int_{t_{0}}^{t} e^{-\int_{t_{0}}^{t} a_{i j}(\xi) d \xi} \\
& \sum_{D^{k l} \in N_{r}(i, j)} D_{i j}^{k l}(s) \sigma_{i j}\left(x_{i j}(s)\right) d w_{i j}(s) .
\end{aligned}
$$

Taking expectations and applying Lemma 2.3, we get

$$
\begin{aligned}
E\left|x_{i j}(t)\right|^{p} \leqslant & 5^{p-1} E\left(\left|\Theta_{i j}^{(1)}\right|^{p}+\left|\Theta_{i j}^{(2)}\right|^{p}\right. \\
& \left.+\left|\Theta_{i j}^{(3)}\right|^{p}+\left|\Theta_{i j}^{(4)}\right|^{p}+\left|\Theta_{i j}^{(5)}\right|^{p}\right),
\end{aligned}
$$

where $i=1,2, \cdots, m, j=1,2, \cdots, n$. Next, we will evaluate every term of (6). For the first term of (6), 
we have

$$
\begin{aligned}
E\left|\Theta_{i j}^{(1)}\right|^{p} & =x_{i j}\left(t_{0}\right) e^{-\int_{t_{0}}^{t} a_{i j}(\xi) d \xi} \\
& \leqslant E\left|x_{i j}\left(t_{0}\right) e^{-\underline{a}_{i j}\left(t-t_{0}\right)}\right|^{p} \\
& \leqslant e^{-p \underline{a}_{i j}\left(t-t_{0}\right)} E\left|x_{i j}\left(t_{0}\right)\right|^{p} .
\end{aligned}
$$

For the second term of (6), we have

$$
\begin{aligned}
& E\left|\Theta_{i j}^{(2)}\right|^{p}=E \mid \int_{t_{0}}^{t} e^{-\int_{t_{0}}^{t} a_{i j}(\xi) d \xi} \\
& \left.\sum_{B^{k l} \in N_{r}(i, j)} B_{i j}^{k l}(s) f_{i j}\left(s, x_{k l}(s)\right) x_{i j}(s) d s\right|^{p} \\
& \leqslant E\left(\int_{t_{0}}^{t} e^{-\underline{a}_{i j}(t-s)} \sum_{B^{k l} \in N_{r}(i, j)} \bar{B}_{i j}^{k l}(s)\left|f_{i j}\left(s, x_{k l}(s)\right)\right|\right. \\
& \left.\times\left|x_{i j}(s)\right| d s\right)^{p} \\
& \leqslant E\left(\int_{t_{0}}^{t} e^{-\underline{a}_{i j}(t-s)} \sum_{B^{k l} \in N_{r}(i, j)} \bar{B}_{i j}^{k l}(s) M_{f}\left|x_{i j}(s)\right| d s\right)^{p} \\
& =E\left(\int_{t_{0}}^{t}\left(e^{-\underline{a}_{i j}(t-s)}\right)^{\frac{p-1}{p}}\left(e^{-\underline{a}_{i j}(t-s)}\right)^{\frac{1}{p}}\right. \\
& \left.\times \sum_{B^{k l} \in N_{r}(i, j)} \bar{B}_{i j}^{k l}(s) M_{f}\left|x_{i j}(s)\right| d s\right)^{p} \\
& \leqslant E\left(\left(\int_{t_{0}}^{t} e^{-\underline{a}_{i j}(t-s)} d s\right)^{p-1} \int_{t_{0}}^{t} e^{-\underline{a}_{i j}(t-s)}\right. \\
& \left.\times\left(\sum_{B^{k l} \in N_{r}(i, j)} \bar{B}_{i j}^{k l}(s) M_{f}\left|x_{i j}(s)\right|\right)^{p} d s\right) \\
& \leqslant\left(\underline{a}_{i j}\right)^{1-p} \int_{t_{0}}^{t} e^{-\underline{a}_{i j}(t-s)}\left(M_{f} \sum_{B^{k l} \in N_{r}(i, j)} \bar{B}_{i j}^{k l}\right)^{p} \\
& \times\left. E|| x_{i j}(s)\right|^{p} d s .
\end{aligned}
$$

For the third term of (6), we have

$$
\begin{aligned}
& E\left|\Theta_{i j}^{(3)}\right|^{p}=E \mid \int_{t_{0}}^{t} e^{-\int_{t_{0}}^{t} a_{i j}(\xi) d \xi} \\
& \left.\sum_{C^{k l} \in N_{r}(i, j)} C_{i j}^{k l}(s) g_{i j}\left(s, x_{k l}\left(s-\tau_{k l}(s)\right)\right) x_{i j}(s) d s\right|^{p}
\end{aligned}
$$

$$
\begin{array}{ll}
= & E\left(\int_{t_{0}}^{t} e^{-\int_{t_{0}}^{t} a_{i j}(\xi) d \xi} \sum_{C^{k l} \in N_{r}(i, j)}\left|C_{i j}^{k l}(s)\right|\right. \\
& \left.\left|g_{i j}\left(s, x_{k l}\left(s-\tau_{k l}(s)\right)\right)\right|\left|x_{i j}(s)\right| d s\right)^{p} \\
\leqslant & E\left(\int_{t_{0}}^{t} e^{-\int_{t_{0}}^{t} a_{i j}(\xi) d \xi} \sum_{C^{k l} \in N_{r}(i, j)} \bar{C}_{i j}^{k l} M_{g}\left|x_{i j}(s)\right| d s\right)^{p} \\
\leqslant & E\left(\left(\int_{t_{0}}^{t} e^{-a_{i j}(t-s)} d s\right)^{p-1} \int_{t_{0}}^{t} e^{-a_{i j}(t-s)}\right. \\
& \left.\times\left(\sum_{C^{k l} \in N_{r}(i, j)}^{p} \bar{C}_{i j}^{k l} M_{g}\left|x_{i j}(s)\right|\right)^{p} d s\right)^{p} \\
\leqslant & \left(\underline{a}_{i j}\right)^{1-p} \int_{t_{0}}^{t} e^{-a_{i j}(t-s)}\left(\sum_{C^{k l} \in N_{r}(i, j)} \bar{C}_{i j}^{k l} M_{g}\right)^{p} \\
& \times E\left|x_{i j}(s)\right|^{p} d s .
\end{array}
$$

For the fourth term of (6), we get

$$
\begin{aligned}
E\left|\Theta_{i j}^{(4)}\right|^{p} & =E\left|\int_{t_{0}}^{t} e^{-\int_{t_{0}}^{t} a_{i j}(\xi) d \xi} L_{i j}(s) d s\right|^{p} \\
& \leqslant E\left|\int_{t_{0}}^{t} e^{-a_{i j}(t-s)} L_{i j}(s) d s\right|^{p} \\
& \leqslant\left(\frac{\bar{L}_{i j}}{\underline{a}_{i j}}\right)^{p} .
\end{aligned}
$$

For the fifth term of (6), we get

$$
\begin{aligned}
& E\left|\Theta_{i j}^{(5)}\right|^{p}=E \mid \int_{t_{0}}^{t} e^{-\int_{t_{0}}^{t} a_{i j}(\xi) d \xi} \\
& \left.\sum_{D^{k l} \in N_{r}(i, j)} D_{i j}^{k l}(s) \sigma_{i j}\left(x_{i j}(s)\right) d w_{i j}(s)\right|^{p} \\
\leqslant & \sigma \mid \int_{t_{0}}^{t}\left(e^{-p \underline{a}_{i j}(t-s)}\right. \\
& \left.\left.\times E\left|\sum_{D^{k l} \in N_{r}(i, j)}\left(D_{i j}^{k l}(s)\right)^{2} \sigma_{i j}^{2}\left(x_{i j}(s)\right)\right|^{\frac{p}{2}}\right)^{\frac{2}{p}} d s\right]^{\frac{p}{2}} \\
\leqslant & \sigma(m n)^{\frac{p}{2}}\left[\int _ { t _ { 0 } } ^ { t } \left(e^{-p \underline{a}_{i j}(t-s)}\right.\right.
\end{aligned}
$$




$$
\begin{aligned}
& \left.\left.\times E\left(\sum_{D^{k l} \in N_{r}(i, j)} \bar{D}_{i j}^{k l}\left|x_{i j}(s)\right|\right)^{p}\right)^{\frac{2}{p}} d s\right]^{\frac{p}{2}} \\
= & \sigma(m n)^{\frac{p}{2}}\left[\int _ { t _ { 0 } } ^ { t } \left(e^{-(p-1) \underline{a}_{i j}(t-s)} e^{-\underline{a}_{i j}(t-s)}\right.\right. \\
& \left.\left.\times E\left(\sum_{D^{k l} \in N_{r}(i, j)} \bar{D}_{i j}^{k l}\left|x_{i j}(s)\right|\right)^{p}\right)^{\frac{2}{p}} d s\right]^{\frac{p}{2}} \\
\leqslant & \sigma(m n)^{\frac{p}{2}}\left(\int_{t_{0}}^{t} e^{-\frac{2 \underline{a}_{i j}(p-1)}{p-2}(t-s)} d s\right)^{\frac{p}{2}-1} \\
& \times\left(\int_{t_{0}}^{t} e^{-\underline{a}_{i j}(t-s)} E\left(\sum_{D^{k l} \in N_{r}(i, j)} \bar{D}_{i j}^{k l}\left|x_{i j}(s)\right|\right)^{p} d s\right) \\
\leqslant & \sigma(m n)^{\frac{p}{2}}\left(\frac{2 \underline{a}_{i j}(p-1)}{p-2}\right)^{1-\frac{p}{2}} \\
& \times\left(\int_{t_{0}}^{t} e^{-\underline{a}_{i j}(t-s)} E\left(\sum_{D^{k l} \in N_{r}(i, j)} \bar{D}_{i j}^{k l}\left|x_{i j}(s)\right|\right)^{p} d s\right) \\
\leqslant & \sigma(m n)^{\frac{p}{2}}\left(\frac{2 \underline{a}_{i j}(p-1)}{p-2}\right)^{1-\frac{p}{2}} \\
& \times\left(\int_{t_{0}}^{t} e^{-\underline{a}_{i j}(t-s)}\left(\sum_{D^{k l} \in N_{r}(i, j)} \bar{D}_{i j}^{k l}\right)^{p}\right. \\
& \left.\times E\left|x_{i j}(s)\right|^{p} d s\right) .
\end{aligned}
$$

It follows from (8)-(11) that

$$
\begin{aligned}
& E\left|x_{i j}(t)\right|^{p} \leqslant 5^{p-1}\left\{e^{-p \underline{a}_{i j}\left(t-t_{0}\right)} E\left|x_{i j}\left(t_{0}\right)\right|^{p}\right. \\
& +\left(\frac{\bar{L}_{i j}}{\underline{a}_{i j}}\right)^{p}+\left(\underline{a}_{i j}\right)^{1-p} \int_{t_{0}}^{t} e^{-\underline{a}_{i j}(t-s)} \\
& \left.\left(M_{f} \sum_{B^{k l} \in N_{r}(i, j)} \bar{B}_{i j}^{k l}\right)^{p} E|| x_{i j}(s)\right|^{p} d s \\
& +\left(\underline{a}_{i j}\right)^{1-p} \int_{t_{0}}^{t} e^{-a_{i j}(t-s)}\left(M_{g} \sum_{C^{k l} \in N_{r}(i, j)} \bar{C}_{i j}^{k l}\right)^{p} \\
& \times E\left|x_{i j}(s)\right|^{p} d s
\end{aligned}
$$

$$
\begin{aligned}
& +\sigma(m n)^{\frac{p}{2}}\left(\frac{2 \underline{a}_{i j}(p-1)}{p-2}\right)^{1-\frac{p}{2}} \\
& \times\left(\int_{t_{0}}^{t} e^{-a_{i j}(t-s)}\left(\sum_{D^{k l} \in N_{r}(i, j)} \bar{D}_{i j}^{k l}\right)^{p}\right. \\
& \left.\left.\times E\left|x_{i j}(s)\right|^{p} d s\right)\right\} .
\end{aligned}
$$

Define

$$
V(t)=\left(v_{11}(t), V_{12}(t), \cdots, V_{m n}(t)\right)^{T},
$$

where $V_{i j}(t)=E\left|x_{i j}(t)\right|^{p}, i=1,2, \cdots, m, j=$ $1,2, \cdots, n$. It follows from (12) that

$V_{i j}(t) \leqslant 5^{p-1} e^{-\delta\left(t-t_{0}\right)} V_{i j}\left(t_{0}\right)+\int_{t_{0}}^{t} e^{-\delta(t-s)} V_{i j}(s) d s+\boldsymbol{l}$,

where $\imath=\max _{(i, j)}\left\{\left(\frac{\bar{L}_{i j}}{\underline{a}_{i j}}\right)^{p}\right\}$. In view of (H3) and Lemma 2.4, we know that the solutions of (1) are $p$-uniformly bounded and the family of all solutions of (1) is $p$-point dissipative. By Lemma 2.2, we can conclude that (1) has an $\omega$-periodic solution. The proof of Theorem 3.1 is completed.

\section{4. $\quad p$-exponential Stability of Periodic Solution}

In this section, we will consider the $p$-exponential stability of periodic solutions of (1).

Theorem 4.1 In addition to (H1)-(H2), assume further that

(H4) there exists an integer $p>2$ such that $\left(\rho_{1}+\right.$ $\left.\rho_{2}\right) \delta^{-1}<1$, where

$$
\begin{aligned}
\rho_{1}= & \max _{(i, j)}\left\{\sigma ^ { p - 1 } \left(( \underline { a } _ { i j } ) ^ { 1 - p } \left[\left(M_{f} \sum_{B^{k l} \in N_{r}(i, j)} \bar{B}_{i j}^{k l}\right)^{p}\right.\right.\right. \\
& \left.+\left(N L_{i j f} \sum_{B^{k l} \in N_{r}(i, j)} \bar{B}_{i j}^{k l}\right)^{p}\right] \\
& +\sigma(m n)^{\frac{p}{2}}\left(\frac{2 \underline{a}_{i j}(p-1)}{p-2}\right)^{1-\frac{p}{2}} \\
& \left.\left.\times\left(\sum_{D^{k l} \in N_{r}(i, j)} \bar{D}_{i j}^{k l}\right)^{p}\right)\right\}
\end{aligned}
$$


and

$$
\begin{aligned}
\rho_{2}= & \max _{(i, j)}\left\{\sigma ^ { p - 1 } ( \underline { a } _ { i j } ) ^ { 1 - p } \left[\left(M_{g} \sum_{C^{k l} \in N_{r}(i, j)} \bar{C}_{i j}^{k l}\right)^{p}\right.\right. \\
& \left.\left.+\left(N L_{i j g} \sum_{C^{k l} \in N_{r}(i, j)} \bar{C}_{i j}^{k l}\right)^{p}\right]\right\},
\end{aligned}
$$

then the periodic solution of (1) is p-exponentially stable.

Proof Obviously, if (H4) holds, then (H3) is fulfilled. In view of Theorem 3.1, we know that (1) has an $\omega$-periodic solution $x^{*}(t)=\left\{x_{i j}^{*}(t)\right\}$ with the initial condition $\varphi(t)=\left\{\varphi_{i j}(t)\right\}, i=1,2, \cdot, m, j=$ $1,2, \cdots, n$. Thus $x^{*}(t)$ is $p$-uniform, namely, there is a constant $C_{0}>0$ such that $E\left|x_{i j}^{*}(t)\right|^{p}<C_{0}, i=$ $1,2, \cdot, m, j=1,2, \cdots, n$. Assume that $x(t)=\left\{x_{i j}(t)\right.$ is an arbitrary solution of (1) with the initial condition $\psi(t)=\left\{\psi_{i j}(t)\right\}, i=1,2, \cdot, m, j=1,2, \cdots, n$. Let

$$
u(t)=\left\{u_{i j}(t)\right\}=\left\{x_{i j}(t)-x_{i j}^{*}(t)\right\}
$$

where $i=1,2, \cdot, m, j=1,2, \cdots, n$. Then for $i=$ $1,2, \cdot, m, j=1,2, \cdots, n$ and $t \geqslant t_{0}$, we get

$$
\begin{aligned}
& d u_{i j}(t)=\left[-a_{i j}(t) u_{i j}(t)\right. \\
& -\sum_{B^{k l} \in N_{r}(i, j)} B_{i j}^{k l}(t)\left(f_{i j}\left(t, x_{k l}(t)\right) x_{i j}(t)\right. \\
& \left.-f_{i j}\left(t, x_{k l}^{*}(t)\right) x_{i j}^{*}(t)\right) \\
& -\sum_{C^{k l} \in N_{r}(i, j)} C_{i j}^{k l}(t)\left(g_{i j}\left(t, x_{k l}\left(t-\tau_{k l}(t)\right)\right) x_{i j}(t)\right. \\
& \left.\left.-g_{i j}\left(t, x_{k l}^{*}\left(t-\tau_{k l}(t)\right)\right) x_{i j}^{*}(t)\right)\right] d t \\
& +\sum_{D^{k l} \in N_{r}(i, j)} D_{i j}^{k l}(t)\left(\sigma_{i j}\left(x_{i j}(t)\right)\right. \\
& \left.-\sigma_{i j}\left(x_{i j}^{*}(t)\right)\right) d w_{i j}(t)
\end{aligned}
$$

with this initial condition

$$
\phi_{i j}(s)=\psi_{i j}(s)-\varphi_{i j}(s), s \in\left[-\tau, t_{0}\right]
$$

where $i=1,2, \cdot, m, j=1,2, \cdots, n$. Applying the method of variation parameter, we have

$$
\begin{aligned}
& u_{i j}(t)=u_{i j}\left(t_{0}\right) e^{-\int_{t_{0}}^{t} a_{i j}(\xi) d \xi}-\int_{t_{0}}^{t} e^{-\int_{t_{0}}^{t} a_{i j}(\xi) d \xi} \\
& \times\left[\sum _ { B ^ { k l } \in N _ { r } ( i , j ) } B _ { i j } ^ { k l } ( s ) \left(f_{i j}\left(s, x_{k l}(s)\right) x_{i j}(s)\right.\right. \\
& \left.-f_{i j}\left(s, x_{k l}^{*}(s)\right) x_{i j}^{*}(s)\right) \\
& +\sum_{C^{k l} \in N_{r}(i, j)} C_{i j}^{k l}(s)\left(g_{i j}\left(s, x_{k l}\left(s-\tau_{k l}(s)\right)\right) x_{i j}(s)\right. \\
& \left.-g_{i j}\left(s, x_{k l}^{*}\left(s-\tau_{k l}(s)\right)\right) x_{i j}^{*}(s)\right] d s \\
& +\int_{t_{0}}^{t} e^{-\int_{t_{0}}^{t} a_{i j}(\xi) d \xi} \sum_{D^{k l} \in N_{r}(i, j)} D_{i j}^{k l}(s)\left(\sigma_{i j}\left(x_{i j}(s)\right)\right. \\
& \left.-\sigma_{i j}\left(x_{i j}^{*}(s)\right)\right) d w_{i j}(s) \\
& =u_{i j}\left(t_{0}\right) e^{-\int_{t_{0}}^{t} a_{i j}(\xi) d \xi}-\int_{t_{0}}^{t} e^{-\int_{t_{0}}^{t} a_{i j}(\xi) d \xi} \\
& \times\left[\sum _ { B ^ { k l } \in N _ { r } ( i , j ) } B _ { i j } ^ { k l } ( s ) \left(f_{i j}\left(s, x_{k l}(s)\right) u_{i j}(s)\right.\right. \\
& \left(f_{i j}\left(s, x_{k l}(s)\right)-f_{i j}\left(s, x_{k l}^{*}(s)\right) x_{i j}^{*}(s)\right) \\
& +\sum_{C^{k l} \in N_{r}(i, j)} C_{i j}^{k l}(s)\left(g_{i j}\left(s, x_{k l}\left(s-\tau_{k l}(s)\right)\right) u_{i j}(s)\right. \\
& +\left(g_{i j}\left(s, x_{k l}\left(s-\tau_{k l}(s)\right)\right)\right. \\
& \left.\left.\left.-g_{i j}\left(s, x_{k l}^{*}\left(s-\tau_{k l}(s)\right)\right)\right) x_{i j}^{*}(s)\right)\right] d s \\
& +\int_{t_{0}}^{t} e^{-\int_{t_{0}}^{t} a_{i j}(\xi) d \xi} \sum_{D^{k l} \in N_{r}(i, j)} D_{i j}^{k l}(s)\left(\sigma_{i j}\left(x_{i j}(s)\right)\right. \\
& \left.-\sigma_{i j}\left(x_{i j}^{*}(s)\right)\right) d w_{i j}(s) \text {, }
\end{aligned}
$$

where $i=1,2, \cdot, m, j=1,2, \cdots, n$ and $t \geqslant t_{0}$. Let

$$
\begin{aligned}
& \Phi_{i j}^{(1)}=u_{i j}\left(t_{0}\right) e^{-\int_{t_{0}}^{t} a_{i j}(\xi) d \xi}, \\
& \Phi_{i j}^{(2)}=\int_{t_{0}}^{t} e^{-\int_{t_{0}}^{t} a_{i j}(\xi) d \xi} \\
& \times \sum_{B^{k l} \in N_{r}(i, j)} B_{i j}^{k l}(s) f_{i j}\left(s, x_{k l}(s)\right) u_{i j}(s) d s, \\
& \Phi_{i j}^{(3)}=\int_{t_{0}}^{t} e^{-\int_{t_{0}}^{t} a_{i j}(\xi) d \xi} \sum_{B^{k l} \in N_{r}(i, j)} B_{i j}^{k l}(s)
\end{aligned}
$$




$$
\begin{aligned}
& \times\left(f_{i j}\left(s, x_{k l}(s)\right)-f_{i j}\left(s, x_{k l}^{*}(s)\right) x_{i j}^{*}(s) d s,\right. \\
& \Phi_{i j}^{(4)}=\int_{t_{0}}^{t} e^{-\int_{t_{0}}^{t} a_{i j}(\xi) d \xi} \\
& \times \sum_{C^{k l} \in N_{r}(i, j)} C_{i j}^{k l}(s) g_{i j}\left(s, x_{k l}\left(s-\tau_{k l}(s)\right)\right) u_{i j}(s) d s, \\
& \Phi_{i j}^{(5)}=\int_{t_{0}}^{t} e^{-\int_{t_{0}}^{t} a_{i j}(\xi) d \xi} \\
& \times \sum_{C^{k l} \in N_{r}(i, j)} C_{i j}^{k l}(s)\left(g_{i j}\left(s, x_{k l}\left(s-\tau_{k l}(s)\right)\right)\right. \\
& \left.\quad-g_{i j}\left(s, x_{k l}^{*}\left(s-\tau_{k l}(s)\right)\right)\right) x_{i j}^{*}(s) d s, \\
& \Phi_{i j}^{(6)}=\int_{t_{0}}^{t} e^{-\int_{t_{0}}^{t} a_{i j}(\xi) d \xi} \\
& \times \sum_{D^{k l} \in N_{r}(i, j)} D_{i j}^{k l}(s)\left(\sigma_{i j}\left(x_{i j}(s)\right)-\sigma_{i j}\left(x_{i j}^{*}(s)\right)\right) d w_{i j}(s) .
\end{aligned}
$$

Taking expectations and applying Lemma 2.3, we have

$$
\begin{aligned}
E\left|u_{i j}(t)\right|^{p} \leqslant & 6^{p-1} E\left(\left|\Phi_{i j}^{(1)}\right|^{p}+\left|\Phi_{i j}^{(2)}\right|^{p}+\left|\Phi_{i j}^{(3)}\right|^{p}\right. \\
& \left.+\left|\Phi_{i j}^{(4)}\right|^{p}+\left|\Phi_{i j}^{(5)}\right|^{p}+\left|\Phi_{i j}^{(6)}\right|^{p}\right) .(18
\end{aligned}
$$

where $i=1,2, \cdots, m, j=1,2, \cdots, n$. Applying the similar method in the proof of Theorem 3.1, we get

$$
\begin{aligned}
E\left|\Phi_{i j}^{(1)}\right|^{p} \leqslant & e^{-p \underline{a}_{i j}\left(t-t_{0}\right)} E\left|u_{i j}\left(t_{0}\right)\right|^{p} \\
E\left|\Phi_{i j}^{(2)}\right|^{p}= & E \mid \int_{t_{0}}^{t} e^{-\int_{t_{0}}^{t} a_{i j}(\xi) d \xi} \\
& \times\left.\sum_{B^{k l} \in N_{r}(i, j)} B_{i j}^{k l}(s) f_{i j}\left(s, x_{k l}(s)\right) u_{i j}(s) d s\right|^{p} \\
\leqslant & \left(\underline{a}_{i j}\right)^{1-p} \int_{t_{0}}^{t} e^{-\underline{a_{i j}(t-s)}}\left(M_{f} \sum_{B^{k l} \in N_{r}(i, j)} \bar{B}_{i j}^{k l}\right)^{p} \\
& \times\left. E|| u_{i j}(s)\right|^{p} d s, \\
E\left|\Phi_{i j}^{(3)}\right|^{p} \leqslant & E \mid \int_{t_{0}}^{t} e^{-\int_{t_{0}}^{t} a_{i j}(\xi) d \xi} \\
& \times \sum_{B^{k l} \in N_{r}(i, j)} B_{i j}^{k l}(s)\left(f_{i j}\left(s, x_{k l}(s)\right)\right. \\
& -\left.f_{i j}\left(s, x_{k l}^{*}(s)\right) x_{i j}^{*}(s) d s\right|^{p}
\end{aligned}
$$

$$
\begin{aligned}
\leqslant & E\left(\int_{t_{0}}^{t} e^{-\underline{a}_{i j}(t-s)}\right. \\
& \left.\times \sum_{B^{k l} \in N_{r}(i, j)} \bar{B}_{i j}^{k l} C_{0} L_{i j f}\left|u_{k l}(s)\right| d s\right)^{p} \\
\leqslant & \left(\underline{a}_{i j}\right)^{1-p} \int_{t_{0}}^{t} e^{-\underline{a}_{i j}(t-s)} \\
& \times\left(C_{0} L_{i j f} \sum_{B^{k l} \in N_{r}(i, j)} \bar{B}_{i j}^{k l}\right)^{p} E\left|u_{k l}(s)\right|^{p} d s,
\end{aligned}
$$$$
E\left|\Phi_{i j}^{(4)}\right|^{p} \leqslant E \mid \int_{t_{0}}^{t} e^{-\int_{t_{0}}^{t} a_{i j}(\xi) d \xi} \sum_{C^{k l} \in N_{r}(i, j)} C_{i j}^{k l}(s)
$$$$
\times\left. g_{i j}\left(s, x_{k l}\left(s-\tau_{k l}(s)\right)\right) u_{i j}(s) d s\right|^{p}
$$$$
\leqslant\left(\underline{a}_{i j}\right)^{1-p} \int_{t_{0}}^{t} e^{-\underline{a}_{i j}(t-s)}
$$$$
\left(M_{g} \sum_{C^{k l} \in N_{r}(i, j)} \bar{C}_{i j}^{k l}\right)^{p} E \|\left. u_{k l}\left(s-\tau_{k l}(s)\right)\right|^{p} d s,
$$$$
E\left|\Phi_{i j}^{(5)}\right|^{p} \leqslant E \mid \int_{t_{0}}^{t} e^{-\int_{t_{0}}^{t} a_{i j}(\xi) d \xi}
$$$$
\sum_{C^{k l} \in N_{r}(i, j)} C_{i j}^{k l}(s)\left(g_{i j}\left(s, x_{k l}\left(s-\tau_{k l}(s)\right)\right)\right.
$$$$
\left.-g_{i j}\left(s, x_{k l}^{*}\left(s-\tau_{k l}(s)\right)\right)\right)\left.x_{i j}^{*}(s) d s\right|^{p}
$$$$
\leqslant\left(\underline{a}_{i j}\right)^{1-p} \int_{t_{0}}^{t} e^{-\underline{a}_{i j}(t-s)}
$$$$
\times\left(C_{0} L_{i j g} \sum_{C^{k l} \in N_{r}(i, j)} \bar{C}_{i j}^{k l}\right)^{p}
$$$$
\times E\left|u_{k l}\left(s-\tau_{k l}(s)\right)\right|^{p} d s,
$$

$$
\begin{aligned}
E\left|\Phi_{i j}^{(6)}\right|^{p} \leqslant & E \mid \int_{t_{0}}^{t} e^{-\int_{t_{0}}^{t} a_{i j}(\xi) d \xi} \\
& \times \sum_{D^{k l} \in N_{r}(i, j)} D_{i j}^{k l}(s)\left(\sigma_{i j}\left(x_{i j}(s)\right)\right. \\
& \left.-\sigma_{i j}\left(x_{i j}^{*}(s)\right)\right)\left.d w_{i j}(s)\right|^{p} \\
\leqslant & \sigma(m n)^{\frac{p}{2}}\left(\frac{2 \underline{a}_{i j}(p-1)}{p-2}\right)^{1-\frac{p}{2}} \\
& \left(\int_{t_{0}}^{t} e^{-\underline{a}_{i j}(t-s)}\left(\sum_{D^{k l} \in N_{r}(i, j)} \bar{D}_{i j}^{k l}\right)^{p}\right.
\end{aligned}
$$




\section{Xu et al. / Existence and p-exponential stability}

$$
\left.\times E\left|u_{i j}(s)\right|^{p} d s\right) .
$$

Then we have

$$
\begin{aligned}
& E\left|u_{i j}\right|^{p} \leqslant 6^{p-1}\left\{e^{-p \underline{a}_{i j}\left(t-t_{0}\right)} E\left|u_{i j}\left(t_{0}\right)\right|^{p}\right. \\
& +\left(\underline{a}_{i j}\right)^{1-p} \int_{t_{0}}^{t} e^{-\underline{a}_{i j}(t-s)}\left(M_{f} \sum_{B^{k l} \in N_{r}(i, j)} \bar{B}_{i j}^{k l}\right)^{p} \\
& \times\left. E|| u_{i j}(s)\right|^{p} d s \\
& +\left(\underline{a}_{i j}\right)^{1-p} \int_{t_{0}}^{t} e^{-\underline{a}_{i j}(t-s)}\left(C_{0} L_{i j f} \sum_{B^{k l} \in N_{r}(i, j)} \bar{B}_{i j}^{k l}\right)^{p} \\
& \times E\left|u_{k l}(s)\right|^{p} d s \\
& +\left(\underline{a}_{i j}\right)^{1-p} \int_{t_{0}}^{t} e^{-\underline{a}_{i j}(t-s)}\left(M_{g} \sum_{C^{k l} \in N_{r}(i, j)} \bar{C}_{i j}^{k l}\right)^{p} \\
& \times\left. E|| u_{k l}\left(s-\tau_{k l}(s)\right)\right|^{p} d s \\
& +\left(\underline{a}_{i j}\right)^{1-p} \int_{t_{0}}^{t} e^{-\underline{a}_{i j}(t-s)}\left(C_{0} L_{i j g} \sum_{C^{k l} \in N_{r}(i, j)} \bar{C}_{i j}^{k l}\right)^{p} \\
& \times E\left|u_{k l}\left(s-\tau_{k l}(s)\right)\right|^{p} d s \\
& +\sigma(m n)^{\frac{p}{2}}\left(\frac{2 \underline{a}_{i j}(p-1)}{p-2}\right)^{1-\frac{p}{2}} \\
& \times\left(\int_{t_{0}}^{t} e^{-\underline{a}_{i j}(t-s)}\left(\sum_{D^{k l} \in N_{r}(i, j)} \bar{D}_{i j}^{k l}\right)^{p}\right. \\
& \left.\left.\times E\left|u_{i j}(s)\right|^{p} d s\right)\right\} \text {. }
\end{aligned}
$$

Define

$$
W(t)=\left(W_{11}(t), W_{12}(t), \cdots, W_{m n}(t)\right)^{T},
$$

where $W_{i j}(t)=E\left|u_{i j}(t)\right|^{p}, i=1,2, \cdots, m, j=$ $1,2, \cdots, n$. It follows from (19) that

$$
\begin{aligned}
W_{i j}(t) \leqslant & 6^{p-1} e^{-\delta\left(t-t_{0}\right)} W_{i j}\left(t_{0}\right) \\
& +\int_{t_{0}}^{t} e^{-\delta(t-s)} \rho_{1} W_{i j}(s) d s \\
& +\int_{t_{0}}^{t} e^{-\delta(t-s)} \rho_{2}\left|W_{i j}(s)\right|_{\tau}^{+} d s
\end{aligned}
$$

where

$$
\begin{aligned}
\rho_{1}= & \max _{(i, j)}\left\{\sigma ^ { p - 1 } \left(( \underline { a } _ { i j } ) ^ { 1 - p } \left[\left(M_{f} \sum_{B^{k l} \in N_{r}(i, j)} \bar{B}_{i j}^{k l}\right)^{p}\right.\right.\right. \\
& \left.+\left(C_{0} L_{i j f} \sum_{B^{k l} \in N_{r}(i, j)} \bar{B}_{i j}^{k l}\right)^{p}\right] \\
& +\sigma(m n)^{\frac{p}{2}}\left(\frac{2 \underline{a}_{i j}(p-1)}{p-2}\right)^{1-\frac{p}{2}} \\
& \left.\left.\times\left(\sum_{D^{k l} \in N_{r}(i, j)} \bar{D}_{i j}^{k l}\right)^{p}\right)\right\}
\end{aligned}
$$

and

$$
\begin{aligned}
\rho_{2}= & \max _{(i, j)}\left\{6 ^ { p - 1 } ( \underline { a } _ { i j } ) ^ { 1 - p } \left[\left(M_{g} \sum_{C^{k l} \in N_{r}(i, j)} \bar{C}_{i j}^{k l}\right)^{p}\right.\right. \\
& \left.\left.+\left(C_{0} L_{i j g} \sum_{C^{k l} \in N_{r}(i, j)} \bar{C}_{i j}^{k l}\right)^{p}\right]\right\} .
\end{aligned}
$$

In view of $(\mathrm{H} 4)$ and Lemma 2.5 , we can conclude that the periodic solution $x^{*}(t)$ of (1) is $p$ exponentially stable. The proof of Theorem 4.1 is completed.

Remark 4.1In Zhao and Zhang ${ }^{27}$, Zhao and Zhang investigated the almost periodic solution of the following shunting inhibitory cellular neural networks with variable coefficients and time-varying delays

$$
\begin{aligned}
& \dot{x}_{i j}(t)=-a_{i j}(t) x_{i j}(t) \\
& -\sum_{B^{k l} \in N_{r}(i, j)} B_{i j}^{k l}(t) f_{i j}\left(t, x_{k l}(t)\right) x_{i j}(t) \\
& -\sum_{C^{k l} \in N_{r}(i, j)} C_{i j}^{k l}(t) g_{i j}\left(t, x_{k l}\left(t-\tau_{k l}(t)\right)\right) x_{i j}(t) \\
& +L_{i j}(t) .
\end{aligned}
$$

By applying Dini derivative, they established some criteria on the existence and local exponential stability of (22). All the results in Zhao and Zhang ${ }^{27}$ can not be applicable to system (1) to obtain the the existence and p-exponential stability of periodic solutions. This implies that the results of this article are essentially new. 


\section{An Example with Its Numerical Simulations}

Example 5.1 Let $i=j=2$. Consider the following stochastic shunting inhibitory cellular neural networks with time-varying delays

$$
\begin{aligned}
& d x_{i j}(t)=\left[-a_{i j}(t) x_{i j}(t)+L_{i j}(t)\right. \\
& -\sum_{B^{k l} \in N_{r}(i, j)} B_{i j}^{k l}(t) f_{i j}\left(t, x_{k l}(t)\right) x_{i j}(t) \\
& \left.-\sum_{C^{k l} \in N_{r}(i, j)} C_{i j}^{k l}(t) g_{i j}\left(t, x_{k l}\left(t-\tau_{k l}(t)\right)\right) x_{i j}(t)\right] d t \\
& +\sum_{D^{k l} \in N_{r}(i, j)} D_{i j}^{k l}(t) \sigma_{i j}\left(x_{i j}(t)\right) d w_{i j}(t),
\end{aligned}
$$

where $f_{i j}(t, x)=\sin \frac{1}{5} x+\frac{4}{5} x, g_{i j}(t, x)=\sin \frac{1}{3} x+\frac{2}{3} x$ and

$$
\begin{aligned}
& {\left[\begin{array}{ll}
a_{11}(t) & a_{12}(t) \\
a_{21}(t) & a_{22}(t)
\end{array}\right]} \\
& =\left[\begin{array}{ll}
0.3+0.02 \cos \frac{\pi}{2} t & 0.4+0.02 \sin \frac{\pi}{2} t \\
0.4+0.01 \cos \frac{\pi}{2} t & 0.3+0.01 \cos \frac{\pi}{2} t
\end{array}\right] \\
& {\left[\begin{array}{ll}
B_{11}(t) & B_{12}(t) \\
B_{21}(t) & B_{22}(t)
\end{array}\right]} \\
& =\left[\begin{array}{cc}
0.02\left|\cos \frac{\pi}{4} t\right| & 0.03 \sin \frac{\pi}{4} t \\
0.01 \sin \frac{\pi}{4} t & 0.02 \cos \frac{\pi}{4} t
\end{array}\right] \\
& {\left[\begin{array}{ll}
C_{11}(t) & C_{12}(t) \\
C_{21}(t) & C_{22}(t)
\end{array}\right]} \\
& =\left[\begin{array}{cc}
0.03\left|\sin \frac{\pi}{4} t\right| & 0.03 \cos \frac{\pi}{4} t \\
0.02 \cos \frac{\pi}{4} t & 0.02 \sin \frac{\pi}{4} t
\end{array}\right] \\
& {\left[\begin{array}{ll}
D_{11}(t) & D_{12}(t) \\
D_{21}(t) & D_{22}(t)
\end{array}\right]} \\
& =\left[\begin{array}{cc}
0.02\left|\cos \frac{\pi}{4} t\right| & 0.01 \sin \frac{\pi}{4} t \\
0.01 \cos \frac{\pi}{4} t & 0.02 \sin \frac{\pi}{4} t
\end{array}\right] \\
& {\left[\begin{array}{ll}
L_{11}(t) & L_{12}(t) \\
L_{21}(t) & L_{22}(t)
\end{array}\right]} \\
& =\left[\begin{array}{ll}
0.2 \cos \frac{\pi}{2} t & 0.1 \sin \frac{\pi}{2} t \\
0.3 \cos \frac{\pi}{2} t & 0.2 \sin \frac{\pi}{2} t
\end{array}\right] \\
& =\left[\begin{array}{ll}
\sigma_{11}(u) & \sigma_{12}(u) \\
\sigma_{21}(u) & \sigma_{22}(u)
\end{array}\right] \\
& =\left[\begin{array}{ll}
0.2 \sin u & 0.2 \sin u \\
0.2 \cos u & 0.1 \sin u
\end{array}\right]
\end{aligned}
$$

$$
\begin{aligned}
& {\left[\begin{array}{ll}
\tau_{11}(t) & \tau_{12}(t) \\
\tau_{21}(t) & \tau_{22}(t)
\end{array}\right]} \\
& =\left[\begin{array}{cc}
0.01|\sin t| & 0.02|\cos u| \\
0.02|\sin u| & 0.01|\cos u|
\end{array}\right] .
\end{aligned}
$$

Then $L_{i j f}=L_{i j g}=M_{f}=M_{g}=1(i, j=1,2), \tau=$ $0.02, L_{11 \sigma}=L_{22 \sigma}=0.1, L_{12 \sigma}=L_{21 \sigma}=0.2$ and

$$
\begin{aligned}
& {\left[\begin{array}{ll}
\sum_{B^{k l} \in N_{1}(1,1)} \bar{B}_{11}^{k l} & \sum_{B^{k l} \in N_{1}(1,2)} \bar{B}_{12}^{k l} \\
\sum_{B^{k l} \in N_{1}(2,1)} \bar{B}_{21}^{k l} & \sum_{B^{k l} \in N_{1}(2,2)} \bar{B}_{22}^{k l}
\end{array}\right]} \\
& =\left[\begin{array}{ll}
0.08 & 0.12 \\
0.04 & 0.08
\end{array}\right], \\
& {\left[\begin{array}{ll}
\sum_{C^{k l} \in N_{1}(1,1)} \bar{C}_{11}^{k l} & \sum_{C^{k l} \in N_{1}(1,2)} \bar{C}_{12}^{k l} \\
\sum_{B^{k l} \in N_{1}(2,1)} \bar{C}_{21}^{k l} & \sum_{C^{k l} \in N_{1}(2,2)} \bar{C}_{22}^{k l}
\end{array}\right]} \\
& =\left[\begin{array}{ll}
0.12 & 0.12 \\
0.08 & 0.08
\end{array}\right], \\
& {\left[\begin{array}{ll}
\underline{a}_{11} & \underline{a}_{12} \\
\underline{a}_{21} & \underline{a}_{22}
\end{array}\right]} \\
& =\left[\begin{array}{ll}
0.28 & 0.38 \\
0.39 & 0.29
\end{array}\right],\left[\begin{array}{ll}
\bar{L}_{11} & \bar{L}_{12} \\
\bar{L}_{21} & \bar{L}_{22}
\end{array}\right] \\
& =\left[\begin{array}{ll}
0.2 & 0.1 \\
0.3 & 0.2
\end{array}\right] .
\end{aligned}
$$

Take $p=3, r=1$. Then we have $\delta \approx 0.2302, \sigma_{1} \approx$ $0.0704, \sigma_{2} \approx 0.0462$. It is easy to check that all the conditions in Theorem 3.1 and Theorem 4.2 are fulfilled. Hence we can conclude that then (23) has a 4-periodic solution, which is 3-exponentially stable. The results are shown in Figs. 1-2.

\section{Conclusions}

In this paper, a class of stochastic shunting inhibitory cellular neural networks with time-varying delays are considered. We establish some sufficient conditions ensuring the existence and $p$-exponential stability of periodic solutions for stochastic shunting inhibitory cellular neural networks with timevarying delays by using integral inequalities. Comparisons between our results and the previous results show that our results complement the earlier publications and are completely new. An example is presented to illustrate our main theoretical findings. Our results play an important key in design- 
ing of shunting inhibitory cellular neural networks. The obtained results show that under some appropriate circumstances, stochastic shunting inhibitory cellular neural networks with time-varying delays can display sustainable periodic oscillatory phenomenon. These periodic oscillatory phenomenon can help us to process visual information quickly and effectively ${ }^{28,29}$. Also periodic oscillatory phenomenon can be helpful for us to predict pathological brain states, which is important to diagnose disease in medical science $\mathrm{e}^{30,31,32}$.

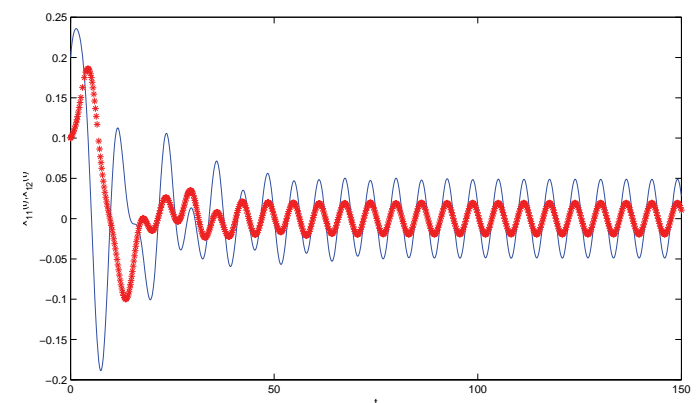

Fig. 1. Transient response of state variables $x_{11}(t)$ and $x_{12}(t)$, where the blue line stands for $x_{11}(t)$ and the red line stands for $x_{12}(t)$.

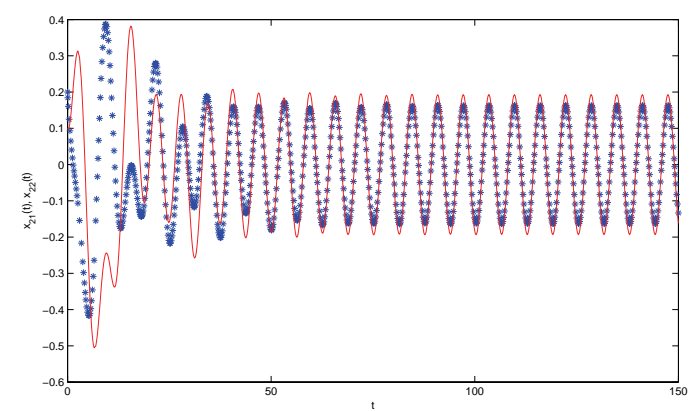

Fig. 2. Transient response of state variables $x_{21}(t)$ and $x_{22}(t)$, where the blue line stands for $x_{21}(t)$ and the red line stands for $x_{22}(t)$.

\section{Acknowledgments}

This work is supported by National Natural Science Foundation of China (No.61673008, No.11261010 and No.11526063), Natural Science and Technology Foundation of Guizhou Province(J[2015]2025 and J[2015]2026), 125 Special Major Science and Technology of Department of Education of Guizhou Province ([2012]011) and Natural Science Foundation of the Education Department of Guizhou Province(KY[2015]482).

\section{References}

1. J. Gao, Q.R. Wang, L.W. Zhang, Existence and stability almost periodic solutions for cellular neural networks with time-varying delays in leakage terms on time scales, Applied Mathematics and Computation, 237 (2014) 639-649.

2. B.W. Liu, J.Y. Shao, Almost periodic solutions for SICNNs with time-varying delays in the leakage terms, Jouranl of Inequalities and Applications, 494 (2013) 1-22.

3. B.W. Liu, Pseudo almost periodic solutions for neutral type CNNs with continuously distributed leakage delays, Neurocomputing, 148 (2015) 445-454.

4. B. Armmar, F. Chérif, A.M. Alimi, Existence and uniqueness of pseudo almost periodic solutions of recurrent neural networks with time-varying coefficients and mixed delays, IEEE Transactions on Neural Networks and Learning Systems, 23 (2012) 109-118.

5. S. Abbas, Y.H. Xia, Almost automorphic solutions of impulsive cellular neural networks with piecewise constant argument, Neural Process Letters, 2014, in press.

6. Y.K. Li, J.Y. Shu, Anti-periodic solutions to impulsive shunting inhibitory cellular neural networks with distributed delays on time scales, Communications in Nonlinear Science and Numerical Simulation, 11 (2011) 137-150.

7. H.H. Wu, Pseudo almost periodic solution of shunting inhibitroy cellular neural networks with delay, Journal of Applied Mathematics, Volume 2011, Article ID 510789, 14 pages.

8. W.T. Wang, B.W. Liu, Global exponential stability of pseudo almost periodic solutions for SICNNs with time-varying leakage delays, Abstract and Applied Analysis, Volume 2014, Article ID 967328, 17 pages.

9. B.W. Liu, Pseudo almost periodic solutions for $\mathrm{CNNs}$ with continuously distributed leakage delays, Neural Process Letters, 42 (2015) 233-256.

10. L.Q. Peng, W.T. Wang, Anti-periodic solutions for shunting inhibitory cellular neural networks with time-varying delays in leakage terms, Neurocomputing, 111 (2013) 27-33.

11. B.W. Liu, New convergence behavior of solutions to shunting inhibitory cellular neural networks with unbounded delays and time-varying coefficients, $A p$ plied Mathematical Modelling, 33 (2009) 54-60. 
12. Y.H. Xia, J.D. Cao, Z.K. Huang, Existence and exponential stability of almost periodic solution for shunting inhibitory cellular neural networks with impulses, Chaos, Solitons \& Fractals, 34 (2007) 1599-1607.

13. B.W. Liu, L.H. Huang, Existence and stability of almost periodic solutions for shunting inhibitory cellular neural networks with time-varying delays, Chaos, Solitons \& Fractals, 31 (2007) 211-217.

14. C.J. Xu, Q.M. Zhang, On anti-periodic solutions of a shunting inhibitory cellular neural networks with distributed delays, Journal of Applied Mathematics and Computing, 47 (2015) 1-13..

15. S. Haykin, Neural Networks, Prentice-Hall, NJ, 1994.

16. S. Blythe, X. Mao, X. Liao, Stability of stochastic delay neural networks, Journal of the Franklin Institute, 338 (2001) 481-495.

17. B. Tojtovska, S. Janković, On some stability problems of impulsive stochastic Cohen-Grossberg neural networks with mixed time delays, Applied Mathematics and Computation, 239 (2014) 211-226.

18. J. Li, M.F. Hu, L.X. Guo, Exponential stability of stochastic memristor-based recurrent neural networks with time-varying delays, Neurocomputing, 138 (2014) 92-98.

19. Y.C. Shi, P.Y. Zhu, Asymptotic stability analysis of stochastic reaction-diffusion Cohen-Grossberg neural networks with mixed time delays, Applied Mathematics and Computation, 242 (2014) 159-167.

20. Q.X. Zhu, J.D. Cao, Mean-square exponential inputto-state stability of stochastic delayed neural networks, Neurocomputing, 131 (2014) 157-163.

21. A. Meyer-Bäse, G. Botella, L.R. Rusinek, Stochastic stability analysis of competitive neural networks with different time-scales, Neurocomputing, 118 (2013) 115-118.

22. D.S. Li, X.H. Wang, D.Y. Xu, Existence and global $p$-exponential stability of periodic solution for impulsive stochastic neural networks with delays, Nonlinear Analysis: Hybrid Systems, 6 (2012) 847-858.

23. R. Khasminskii, Stochastic Stability of Differential
Equations, Stochastic Modelling and Applied Probability, vol. 66. Springer, Berlin, 2012.

24. D.Y. Xu, Y.M. Huang, Z.G. Yang, Existence theorems for periodic Markov process and stochastic functional differential equations, Discrete and Continuous Dynamical Systems, 24 (2009) 1005-1023.

25. F.K. Wu, S.G. Hu, Y. Liu, Positive solution and its asymptotic behavior of stochastic functional Kolmogorov-type system, Journal of Mathematical Analysis and Applications, 364 (2010) 104-118.

26. L. Yang, Y.K. Li, Periodic solutions for stochastic shunting inhibitory cellular neural networks with distributed delays, Advances in Difference Equations, 37 (2014) d1-14.

27. W.R. Zhao, H.S. Zhang, On almost periodic solution of shunting inhibitory cellular neural networks with variable coefficients and time-varying delays, Nonlinear Analysis: Real World Applications, 9 (2008) 23262336.

28. Y.F. Weng, L. Ju, J. Wang, Cellular neural networks and Biological visual information processing model, Journal of Beijing Technology and Business University (Natural Science Edition), 25 (2007) 42-58.

29. C.J. Xu, Q.M. Zhang, Y.S. Wu, Existence and stability of pseudo almost periodic solutions for shunting inhibitory cellular neural networks with neutral type delays and time-varying leakage delays, Network: Computation in Neural Systems, 25 (2014) 168-192.

30. J. TØnesen, Optogenetic cell control in experimental models of neurological disorders, Behavioural Brain Research, 255 (2013) 35-43.

31. P. Gerlee, A.R.A. Anderson, Modelling evolutionary cell behaviour using neural networks: Application to tumour growth, Biosystems, 95 (2009) 166-174.

32. M. Moghtadaei, M.R.H, Golpayegani, R. Malekzadeh, A variable structure fuzzy neural network model of squamous dysplasia and esophageal squamous cell carcinoma based on a global chaotic optimization algorithm. Journal of Theoretical Biology, 318 (2013) 164-172. 